Proyecciones Journal of Mathematics

Vol. 35, No 4, pp. 491-503, December 2016.

Universidad Católica del Norte

Antofagasta - Chile

\title{
About the solutions of linear control systems on Lie groups
}

\author{
Víctor Ayala * \\ Universidad de Tarapacá, Chile \\ Adriano Da Silva ${ }^{\dagger}$ \\ Universidade Estadual de Campinas, Brasil \\ and \\ Eyüp Kizil \\ Universidade de Sao Paulo, Brasil \\ Received: July 2016. Accepted : September 2016
}

\begin{abstract}
In this paper we prove in details the completeness of the solutions of a linear control system on a connected Lie group. On the other hand, we summarize some results showing how to compute the solutions. Some examples are given.
\end{abstract}

Keywords : Linear control systems, Lie groups, solutions.

AMS 2010 subject classification : 16W25; 93B05; $93 C 05$.

\footnotetext{
*Supported by Proyecto Fondecyt No. 1150292, Conicyt.

${ }^{\dagger}$ Supported by Fapesp Grant No. 2016/11135-2.

${ }^{\ddagger}$ Supported by Tubitak Grant No. 116C081.
} 


\section{Introduction}

In [1] Ayala and Tirao introduced the concept of linear control system $\Sigma$ on a connected Lie group $G$ as the family of ordinary differential equations given by

$$
\Sigma: \quad \dot{g}(t)=\mathcal{X}(g(t))+\sum_{j=1}^{m} u_{j}(t) X^{j}(g(t))
$$

where $\mathcal{X}$ is a linear vector field, (see Definition 2.1), $X^{j}$ are right-invariant vector fields for $j=1, \ldots, m$ and $u=\left(u_{1}, \ldots, u_{m}\right)$ belongs to the class of admissible control functions $\mathcal{U} \subset L^{\infty}\left(\mathbf{R}, \mathbf{R}^{m}\right)$.

Linear control systems are important for at least two reasons. First, they are a natural generalization of the well known linear control system on the Euclidean space $G=\mathbf{R}^{n}$ given by

$\dot{x}(t)=A x(t)+B u(t), \quad A \in \mathbf{R}^{n \times n}, B \in \mathbf{R}^{n \times m}$ and $u=\left(u_{1}, \ldots, u_{m}\right) \in \mathcal{U}$.

Besides that, [9] Jouan proved that $\Sigma$ is relevant from theoretical and practical point of view, see also [2], [3], [4], [6], [7] and [8]. Actually, he shows that any general affine control system

$$
\Sigma_{M}: \quad \dot{x}(t)=X(x(t))+\sum_{j=1}^{m} u_{j}(t) X^{j}(x(t)),
$$

on a connected manifold $M$ whose dynamics generate a finite dimensional Lie algebra, i.e.

$$
\operatorname{dim} \operatorname{Span}_{\mathcal{L A} \mathcal{A}}\left\{X, Y^{1}, \ldots, Y^{m}\right\}<\infty
$$

is equivalent to a linear control system on a Lie group $G$ or on a homogeneous space of $G$.

In this paper we prove in details the completeness of the $\Sigma$-solution $\phi(t, g, u)$ for any initial condition $g \in G$, control $u \in \mathcal{U}$ and $t \in \mathbf{R}$. Furthermore, we show a way to compute the flow of an arbitrary drift $\mathcal{X}$ and then the solution $\phi(t, g, u)$, which is specially suitable for nilpotent and simply connected Lie groups. In the same spirit we also recall a series solution-formula which appears in [1]. 


\section{Linear vector fields}

In [1] the authors introduced the notion of the normalizer of a Lie algebra $\mathrm{g}$ which is by definition the space

$$
\eta:=\operatorname{norm}_{\mathcal{X}}(G)(\mathbf{g}):=\{F \in \mathcal{X}(\mathcal{G}) ; \text { for all } \mathcal{Y} \in \mathbf{g}, \quad[\mathcal{F}, \mathcal{Y}] \in \mathbf{g}\}
$$

where $\mathcal{X}(\mathcal{G})$ stands for the set of all smooth vector fields on $G$.

Let us denote by $e \in G$ the identity element of $G$.

Definition: 2.1. A vector field $\mathcal{X}$ on $G$ is said to be linear if it belongs to $\eta$ and $\mathcal{X}(e)=0$.

The following result (Theorem 1 of [7]) gives equivalent conditions for a vector field on $G$ to be linear.

Theorem: 2.2. Let $\mathcal{X}$ be a vector field on a connected Lie group $G$. The following conditions are equivalent:

1. $\mathcal{X}$ is linear

2. $\mathcal{X}$ is an infinitesimal automorphism

3. $\mathcal{X}$ satisfies

$$
\mathcal{X}(g h)=\left(d L_{g}\right)_{h} \mathcal{X}(h)+\left(d R_{h}\right)_{g} \mathcal{X}(g), \text { for all } g, h \in G .
$$

If we denote by $\left(\varphi_{t}\right)_{t \in \mathbf{R}}$ the flow associated to the linear vector field $\mathcal{X}$, by definition an infinitesimal automorphism is a vector field such that its flow

$$
\left\{\varphi_{t}: t \in \mathbf{R}\right\} \subset \operatorname{Aut}(G)
$$

is a subgroup of the Lie group of the automorphism of $G$. As usual, $L_{g}$ and $R_{g}$ denote the left and right translations on $G$ and $d L_{g}, d R_{g}$ their derivatives.

Remark: 2.3. Item 2. of Theorem 2.2 shows that $\mathcal{X}$ is complete. In fact, since $\mathcal{X}(e)=0$ for $t \in \mathbf{R}$ it follows that $\varphi_{t}$ is well defined in a neighborhood $V_{t}$ of $e \in G$. Since $G$ is connected, $V_{t}$ generates $G$. So, for any $g \in G$ there exist $g_{1}, \ldots, g_{n} \in V_{t}$ such that $g=g_{1} \cdots g_{n}$ implying that

$$
\varphi_{t}(g)=\varphi_{t}\left(g_{1} \cdots g_{n}\right)=\varphi_{t}\left(g_{1}\right) \cdots \varphi_{t}\left(g_{n}\right)
$$

is well defined and therefore $\mathcal{X}$ is complete. 
Next we show that associated with any linear vector field $\mathcal{X}$ there exists a $\mathbf{g}$-derivation that is related with the flow of $\mathcal{X}$. Recall that $D: \mathbf{g} \rightarrow \mathbf{g}$ is a derivation if

$$
D[X, Y]=[D X, Y]+[X, D Y], \text { for any } X, Y \in \mathbf{g} .
$$

Of course $\varphi_{t}(e)=e$ for all $t \in \mathbf{R}$, so if $Y \in \mathbf{g}$ we obtain

$$
[\mathcal{X}, Y](e)=\left(\frac{d}{d t}\right)_{t=0}\left(d \varphi_{-t}\right)_{\varphi_{t}(e)} Y\left(\varphi_{t}(e)\right)=\left(\frac{d}{d t}\right)_{t=0}\left(d \varphi_{-t}\right)_{e} Y(e)
$$

The vector field $Y$ is right invariant, therefore at any point $g \in G$

$$
\begin{gathered}
{[\mathcal{X}, Y](g)=\left(\frac{d}{d t}\right)_{t=0}\left(d \varphi_{-t}\right)_{\varphi_{t}(g)} Y\left(\varphi_{t}(g)\right)=\left(\frac{d}{d t}\right)_{t=0}\left(d \varphi_{-t}\right)_{\varphi_{t}(g)}\left(d R_{\varphi_{t}(g)}\right)_{e} Y(e)} \\
=\left(\frac{d}{d t}\right)_{t=0}\left(d R_{g}\right)_{e}\left(d \varphi_{-t}\right)_{e} Y(e)=\left(d R_{g}\right)_{e}[\mathcal{X}, Y](e) .
\end{gathered}
$$

In fact for any $t \in \mathbf{R}, \varphi_{t}$ is an automorphism of $G$ therefore

$$
\varphi_{-t} \circ R_{\varphi_{t}(g)}=R_{g} \circ \varphi_{-t} .
$$

Thus, for a given linear vector field $\mathcal{X}$, one can associate the derivation $\mathcal{D}$ of $\mathbf{g}$ defined as

$$
\mathcal{D} Y=-[\mathcal{X}, Y](e), \text { for all } Y \in \mathbf{g} .
$$

The minus sign in the above formula comes from the fact $[A x, b]=-A b$ in $\mathbf{R}^{d}$. It is also used in order to avoid a minus sign in the equality stated in Proposition 2 of [7].

Remark: 2.4. For all $t \in \mathbf{R},\left(d \varphi_{t}\right)_{e}=e^{t \mathcal{D}}$. In particular, from the commutative diagram

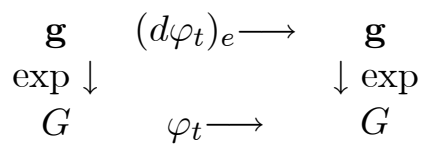

it follows that

$$
\varphi_{t}(\exp Y)=\exp \left(d \varphi_{t}\right)_{e} Y=\exp \left(e^{t \mathcal{D}} Y\right), \text { for all } t \in \mathbf{R}, Y \in \mathbf{g} .
$$


Remark: 2.5. If $G$ is a connected and simply connected nilpotent Lie group the exponential map is a diffeomorphism. In particular, given a derivation it is possible to explicitly compute the drift $\mathcal{X}$ through the formula above via the logarithm map, as follows. Let $\log (g)=Y$, then

$$
\varphi_{t}(g)=\exp \left(e^{t \mathcal{D}} \log (g)\right), \text { for all } t \in \mathbf{R}, g \in G
$$

Remark: 2.6. In [1] the authors proved that for connected and simply connected Lie groups the normalizer is isomorphic to the semidirect product $\mathbf{g} \otimes_{s} \partial \mathbf{g}$ between $\mathbf{g}$ and the Lie algebra of derivations $\partial \mathbf{g}$ of $\mathbf{g}$. Therefore, any linear vector field defines a derivation, but the converse is true just for simply connected Lie groups.

Remark: 2.7. For a general Lie algebra $\mathbf{g}$ pick an inner derivation $D \in \partial \mathbf{g}$ which means $D=[\cdot, Y]$, where $Y \in \mathbf{g}$. In this situation $D$ defines a linear vector field $\mathcal{X}=\mathcal{X}^{D}$ and it is easy to determine

$$
\mathcal{X}=\left(\frac{d}{d t}\right)_{t=0} \varphi_{t}
$$

through the computation of its flow as follows

$$
\varphi_{t}(g)=\exp (t Y) g \exp (-t Y), \text { for all } t \in \mathbf{R} .
$$

\section{Completeness of the solutions}

Consider a linear control system on a Lie group $G$ introduced in [1] as

$$
\Sigma: \quad \dot{g}(t)=\mathcal{X}(g(t))+\sum_{j=1}^{m} u_{j}(t) X^{j}(g(t)),
$$

where the drift $\mathcal{X}$ is a linear vector field, $X^{j}$ are right-invariant vector fields, $u \in \mathcal{U} \subset L^{\infty}\left(\mathbf{R}, \Omega \subset \mathbf{R}^{m}\right)$ is the class of admissible controls, with $\Omega \subset \mathbf{R}^{m}$ a convex subset satisfying $0 \in$ int $\Omega$.

Since all the vector fields involved are analytical, for each control function $u \in \mathcal{U}$ and each initial value $g \in G$ there exists a unique solution $\phi(t, g, u)$ defined on an open interval containing $t=0$ and satisfying $\phi(0, g, u)=g$. Note that in general $\phi(t, g, u)$ is just a solution in the sense of Carathéodory, i.e., a locally absolutely continuous curve satisfying the 
corresponding differential equation almost everywhere. In its domain we know that any solution of $\Sigma$ satisfies the cocycle property

$$
\phi(t+s, g, u)=\phi\left(t, \phi(s, g, u), \Theta_{s} u\right)
$$

where the map $\Theta_{t}$ is the shift flow on $\mathcal{U}$ defined by

$$
\left(\Theta_{t} u\right)(s):=u(t+s) .
$$

In the sequel, instead of $\phi(t, g, u)$ we usually write $\phi_{t, u}(g)$. Note that smoothness of the vector fields $\mathcal{X}, X^{1}, \ldots, X^{m}$ implies the smoothness of $\phi_{t, u}$. Moreover, for a fixed $t$ and $u$ the map $g \in G \mapsto \phi_{t, u}(g) \in G$ is a diffeomorphism whose inverse is given by $g \in G \mapsto \phi_{-t, \Theta_{t} u}(g) \in G$.

The next result shows that in order to compute solutions of $\Sigma$ starting from an arbitrary initial condition it is enough to compute the corresponding solution at the identity element.

Proposition: 3.1. For a given $u \in \mathcal{U}, t \in \mathbf{R}$, let us denote by $\phi_{t, u}:=$ $\phi_{t, u}(e)$ the solution of $\Sigma$ starting at the origin $e \in G$. Then, the solution of $\Sigma$ starting at $g \in G$ satisfies

$$
\phi(t, g, u)=\phi_{t, u} \cdot \varphi_{t}(g)=L_{\phi_{t, u}}\left(\varphi_{t}(g)\right) .
$$

Proof: Let us consider the curve $\alpha(t)$ given by

$$
\alpha(t)=\phi_{t, u} \cdot \varphi_{t}(g)
$$

Therefore, $\alpha(0)=g$ and

$$
\begin{aligned}
& \dot{\alpha}(t)=\left(d L_{\phi_{t, u}}\right)_{\varphi_{t}(g)} \frac{d}{d t} \varphi_{t}(g)+\left(d R_{\varphi_{t}(g)}\right)_{\phi_{t, u}} \frac{d}{d t} \phi_{t, u} \\
& =\left(\mathrm{dL}_{\phi_{t, u}}\right)_{\varphi_{t}(g)} \mathcal{X}\left(\varphi_{t}(g)\right)+\left(d R_{\varphi_{t}(g)}\right)_{\phi_{t, u}}\left\{\mathcal{X}\left(\phi_{t, u}\right)+\sum_{j=1}^{m} u_{j}(t) X^{j}\left(\phi_{t, u}\right)\right\} \\
& =\left\{\left(d L_{\phi_{t, u}}\right)_{\varphi_{t}(g)} \mathcal{X}\left(\varphi_{t}(g)\right)+\left(d R_{\varphi_{t}(g)}\right)_{\phi_{t, u}} \mathcal{X}\left(\phi_{t, u}\right)\right\}+\sum_{j=1}^{m} u_{j}(t) X^{j}(\alpha(t)) .
\end{aligned}
$$

By item 2. of Theorem 2.2

$$
\left(d L_{\phi_{t, u}}\right)_{\varphi_{t}(g)} \mathcal{X}\left(\varphi_{t}(g)\right)+\left(d R_{\varphi_{t}(g)}\right)_{\phi_{t, u}} \mathcal{X}\left(\phi_{t, u}\right)=\mathcal{X}\left(\phi_{t, u} \varphi_{t}(g)\right)=\mathcal{X}(\alpha(t))
$$

Consequently 


$$
\dot{\alpha}(t)=\mathcal{X}(\alpha(t))+\sum_{j=1}^{m} u_{j}(t) X^{j}(\alpha(t)) .
$$

By the uniqueness of the solution, we have the desired conclusion.

Theorem: 3.2. For each $u \in \mathcal{U}$ and $g \in G$ the corresponding solution $\phi_{t, u}(g)$ of $\Sigma$ is defined in the whole real line.

Proof: Since the solution of $\Sigma$ starting at $g$ and control $u$ is given by $\phi_{t, u}(g)=\phi_{t, u} \varphi_{t}(g)$ we only have to show that the solution starting at the identity element $e \in G$ is defined for any $t \in \mathbf{R}$.

Consider $u \in \mathcal{U}$ and let $\alpha(t)$ defined on $\left(-\tau^{\prime}, \tau\right)$ be the maximal solution of $\Sigma$ associated with $u$ satisfying $\alpha(0)=e$. Let $\beta(t)$ be also a solution associated with $u$ satisfying $\beta(\tau)=e$ and defined on $(\tau-\delta, \tau+\delta)$. Consider the curve

$$
\gamma(t):=\left\{\begin{array}{cc}
\alpha(t) & t \in\left(-\tau^{\prime}, \tau-\frac{1}{2} \delta\right) \\
\beta(t) \varphi_{t-\left(\tau-\frac{1}{2} \delta\right)}\left(g^{-1} h\right) & {\left[\tau-\frac{1}{2} \delta, \tau+\delta\right)}
\end{array}\right.
$$

where $g=\alpha\left(\tau-\frac{1}{2} \delta\right)$ and $h=\beta\left(\tau-\frac{1}{2} \delta\right)$. It is straightforward to check that $\gamma$ is well defined and continuous. Moreover, for all $t \in\left(-\tau^{\prime}, \tau-\frac{1}{2} \delta\right)$, $\gamma(t)$ it is a solution of $\Sigma$. If we denote by $\eta(t):=\varphi_{t-\left(\tau-\frac{1}{2} \delta\right)}\left(g^{-1} h\right)$ we have, for all $t \in\left[\tau-\frac{1}{2} \delta, \tau+\delta\right)$

$$
\dot{\gamma}(t)=\frac{d}{d t} \beta(t) \eta(t)=\left(d L_{\beta(t)}\right)_{\eta(t)} \dot{\eta}(t)+\left(d R_{\eta(t)}\right)_{\beta(t)} \dot{\beta}(t) .
$$

However,

$$
\dot{\eta}(t)=\mathcal{X}(\eta(t)) \quad \text { and } \quad \dot{\beta}(t)=\mathcal{X}(\beta(t))+\sum_{j=1}^{m} X^{j}(\beta(t))
$$

and so

$$
\begin{gathered}
\eta(t)=\left(d L_{\beta(t)}\right)_{\eta(t)} \mathcal{X}(\eta(t))+\left(d R_{\eta(t)}\right)_{\beta(t)}\left(\mathcal{X}(\beta(t))+\sum_{j=1}^{m} X^{j}(\beta(t))\right) \\
=\left(d L_{\beta(t)}\right)_{\eta(t)} \mathcal{X}(\eta(t))+\left(d R_{\eta(t)}\right)_{\beta(t)} \mathcal{X}(\beta(t))+\sum_{j=1}^{m} X^{j}(\beta(t) \eta(t))
\end{gathered}
$$




$$
=\mathcal{X}(\gamma(t))+\sum_{j=1}^{m} X^{j}(\gamma(t))
$$

showing that $\gamma(t)$ is a solution of $\Sigma$ defined on $\left(-\tau^{\prime}, \tau+\delta\right)$ associated with $u \in \mathcal{U}$ and satisfying $\gamma(0)=e$ which is a contradiction with the maximality of $\alpha(t)$. It turns out that $\alpha$ must be defined in $\left(-\tau^{\prime}, \infty\right)$. Analogously it is possible to show the same for negative times. Thus, the solutions of $\Sigma$ starting at $e \in G$ are defined in the whole real line.

\section{Solution and series}

In this section we recall a result that appears in [1] which shows how to compute the $\Sigma$ solutions when you know the flow of the drift.

Theorem: 4.1. Let us consider a constant admissible control, $u \in \mathbf{R}^{m}$. Therefore, the vector field $\mathcal{X}+\sum_{j=1}^{m}$ has the solution given by

$$
\phi(t, g, u)=\varphi_{t}(x) \exp \left(\sum_{j=1}^{\infty}(-1)^{n+1} t^{n} d_{n}\left(X^{u}, D\right)\right)
$$

where $X^{u}=\sum_{j=1}^{m} u_{j} X^{j} \in \mathbf{g}$ and for each $n \geq 1$,

$$
d_{n}: \mathbf{g} \otimes_{s} \partial \mathbf{g} \longrightarrow \mathbf{g}
$$

is a homogeneous polynomial map of degree $n$.

In particular, some of the first terms of $d_{n}$ are obtained by recursive formula as follows:

$$
\begin{aligned}
d_{1}\left(Y^{u}, D\right) & =Y^{u} \\
d_{2}\left(Y^{u}, D\right) & =\frac{1}{2} D\left(Y^{u}\right) \\
d_{3}\left(Y^{u}, D\right) & =\frac{1}{12}\left[Y^{u}, D\left(Y^{u}\right)\right]+\frac{1}{6} D^{2}\left(Y^{u}\right) \\
d_{4}\left(Y^{u}, D\right) & =\frac{1}{24}\left[Y^{u}, D^{2}\left(Y^{u}\right)\right]+\frac{1}{24} D^{3}\left(Y^{u}\right), \text { etc. }
\end{aligned}
$$




\section{Examples}

In order to build examples of linear control systems on a Lie group $G$ it is worth to compute first the Lie algebra $\partial \mathbf{g}$ of $\mathbf{g}$, see [5]. Of course, the dimension of $\partial \mathbf{g}$ varies from Abelian to semisimple Lie groups. In fact, in the Euclidean case any linear transformation $D: \mathbf{R}^{d} \rightarrow \mathbf{R}^{d}$ is trivially a derivation. However, in a semisimple Lie group every derivation is inner. Thus, the dimension of $\partial \mathbf{g}$ varies from $d^{2}$ to $d$.

In this section we show some examples

Example: 5.1. Let $G=E(2)$ the Lie group of the Euclidean motions of the plane

$$
G=\left\{g=\left(\begin{array}{ccc}
1 & 0 & 0 \\
x & a & b \\
y & -b & \alpha
\end{array}\right):(x, y) \in \mathbf{R}, a^{2}+b^{2}=1\right\} .
$$

Any point $(x, y)$ in $\mathbf{R}^{2}$ is both translated and rotated by the action of elements in $G$. The Lie algebra $\mathbf{g}$ of $G$ is given by

$$
g=\left\{\left(\begin{array}{ccc}
0 & 0 & 0 \\
a & 0 & c \\
b & -c & 0
\end{array}\right): a, b, c \in \mathbf{R}\right\} .
$$

Let us consider the basis

$$
\mathbf{g}=\operatorname{Span}\left\{Y^{1}=\left(\begin{array}{lll}
0 & 0 & 0 \\
1 & 0 & 0 \\
0 & 0 & 0
\end{array}\right), Y^{2}=\left(\begin{array}{lll}
0 & 0 & 0 \\
0 & 0 & 0 \\
1 & 0 & 0
\end{array}\right), Y^{3}=\left(\begin{array}{ccc}
0 & 0 & 0 \\
0 & 0 & 1 \\
0 & -1 & 0
\end{array}\right)\right\}
$$

and the inner derivations $D_{1}$ and $D_{3}$ determined by $Y^{1}$ and $Y^{3}$ respectively. We obtain linear vector fields $\mathcal{X}^{1}=\mathcal{X}^{D_{1}}$ and $\mathcal{X}^{3}=\mathcal{X}^{D_{3}}$ as follows

$$
\begin{aligned}
\mathcal{X}^{1}(g) & =\left(\frac{d}{d t}\right)_{t=0} \exp \left(t Y^{1}\right) g \exp \left(-t Y^{1}\right) \\
& =\left(\frac{d}{d t}\right)_{t=0}\left(\begin{array}{ccc}
1 & 0 & 0 \\
x+t-a t & a & b \\
y+b t & -b & -a
\end{array}\right)=\left(\begin{array}{ccc}
0 & 0 & 0 \\
1-a & 0 & 0 \\
b & 0 & 0
\end{array}\right)
\end{aligned}
$$

and 


$$
\begin{aligned}
\mathcal{X}^{3}(g) & =\left(\frac{d}{d t}\right)_{t=0} \exp \left(t Y^{3}\right) g \exp \left(-t Y^{3}\right) \\
& =\left(\frac{d}{d t}\right)_{t=0}\left(\begin{array}{ccc}
1 & 0 & 0 \\
x \cos t+y \sin t & a & b \\
-x \sin t+y \cos t & -b & a
\end{array}\right)=\left(\begin{array}{ccc}
0 & 0 & 0 \\
y & 0 & 0 \\
-x & 0 & 0
\end{array}\right)
\end{aligned}
$$

Example: 5.2. Let $\mathbf{g}=\mathbf{R} X^{1}+\mathbf{R} X^{2}+\mathbf{R} X^{3}$ the Lie algebra of the connected and simply connected Heisenberg Lie group $G$ with the following generators

$$
X^{1}=\frac{\partial}{\partial x_{1}}, X^{2}=x_{3} \frac{\partial}{\partial x_{1}}+\frac{\partial}{\partial x_{2}} \text { and } X^{3}=\frac{\partial}{\partial x_{3}}
$$

The only one non-vanishing Lie bracket is $\left[X^{3}, X^{2}\right]=X^{1}$. The group $G$ is diffeomorphic to $\mathbf{R}^{3}$ with the non-Abelian group operation $*: G \rightarrow G$ given by

$$
\begin{gathered}
\left(x_{1}, x_{2}, x_{3}\right) *\left(y_{1}, y_{2}, y_{3}\right)=\left(x_{1}+y_{1}+x_{3} y_{2}, x_{2}+y_{2}, x_{3}+y_{3}\right), \text { and } \\
\left(x_{1}, x_{2}, x_{3}\right)^{-1}=\left(-x_{1}+x_{2} x_{3},-x_{2},-x_{3}\right) .
\end{gathered}
$$

On the other hand, the exponential and logarithm maps are given by

$$
\exp \left(a_{1} X^{1}+a_{2} X^{2}+a_{3} X^{3}\right)=\left(a_{1}+\frac{1}{2} a_{2} a_{3}, a_{2}, a_{3}\right)
$$

and

$$
\log \left(x_{1}, x_{2}, x_{3}\right)=\left(x_{1}-\frac{1}{2} x_{2} x_{3}\right) X^{1}+x_{2} X^{2}+x_{3} X^{3} .
$$

Let us consider the linear control system $\Sigma$ given by

$$
\dot{g}=\mathcal{X}(g)+u X^{2}(g), u \in \mathbf{R},
$$

where $g=\left(x_{1}, x_{2}, x_{3}\right) \in G$ and the infinitesimal automorphism $\mathcal{X}$ is determined by

$$
\mathcal{X}_{t}\left(x_{1}, x_{2}, x_{3}\right)=\left(x_{1}+x_{2} t+\frac{1}{2} x_{2}^{2} t, x_{2}, t x_{2}+x_{3}\right) \text {. }
$$


In fact, it can be checked that $\mathcal{X}_{t} \in \operatorname{Aut}(G)$ for every real number $t$. In coordinates, the system $\Sigma$ reads

$\Sigma:\left\{\begin{array}{l}\dot{x}_{1}=x_{2}+\frac{1}{2} x_{2}^{2}+u x_{3} \\ \dot{x}_{2}=u \\ \dot{x}_{3}=x_{2}\end{array}\right.$

According to our previous results, the solution exists and is given by the series-solution as

$$
\phi_{t, u}(g)=\varphi_{t}(g) \exp \left(\sum_{j=1}^{\infty}(-1)^{n+1} t^{n} d_{n}\left(u Y^{2}, D\right)\right)
$$

The derivation $D$ associated to $\mathcal{X}$ is the matrix $\left(\begin{array}{lll}0 & 1 & 0 \\ 0 & 0 & 0 \\ 0 & 1 & 0\end{array}\right)$. Since $D$ is nilpotent with nilpotency degree 2 , it follows that $d_{n}$ is zero for $n \geq 4$. The non-null terms of the series are listed below:

$$
\begin{aligned}
& d_{1}=u Y^{2}, \\
& d_{2}=\frac{1}{2} u\left(Y^{1}+Y^{3}\right), \\
& d_{3}=-\frac{1}{12} u^{2} Y^{1} \text { and } \\
& d_{4}=d_{5}=\cdots=0 .
\end{aligned}
$$

In such a case, we get a finite series

$$
\zeta(t)=t d_{1}-t^{2} d_{2}+t^{3} d_{3}
$$

so that

$$
\exp \zeta(t)=\exp \left(\left(-\frac{t^{3}}{12} u^{2}-\frac{t^{2}}{2} u\right) Y^{1}+u t Y^{2}-\frac{t^{2}}{2} u Y^{3}\right)
$$

By the exponential rule, the solution $\phi_{t, u}(g)$ with initial condition $g$ and constant control $u$ reads

$$
\begin{aligned}
& \phi(t, g, u)= \\
& \left(x_{1}+\left(x_{2}+\frac{1}{2} x_{2}^{2}+u x_{3}\right) t+\left(u x_{2}-\frac{u}{2}\right) t^{2}-\frac{t^{3}}{3} u^{2}, x_{2}+u t, t x_{2}+x_{3}-\frac{t^{2}}{2} u\right) .
\end{aligned}
$$




\section{References}

[1] Ayala, V. and Tirao, J. Linear control systems on Lie groups and controllability, Eds. G. Ferreyra et al., Amer. Math. Soc., Providence, RI, (1999).

[2] Ayala, V. and San Martin, L., Controllability properties of a class of control systems on Lie groups. Lectures Notes in Control and Information Science, (2001).

[3] Ayala, V. and Da Silva, A., Controllability of linear systems on Lie groups with finite semisimple center. Submitted to SIAM Journal, (2016).

[4] Ayala, V, and Silva, A., Control sets of linear systems on Lie groups. Submitted to Nonlinear Differential Equations and Applications, (2016).

[5] Ayala, V, Kizil, E. and Tribuzy, I., On an algoritm for finding derivations of Lie algebras. Proyecciones Mathematical Journal, Vol 31, No. 1, pp. 81-90, (2012.)

[6] Da Silva, A., Controllability of linear systems on solvable Lie groups, SIAM Journal on Control and Optimization, Vol 54, No. 1, pp. 372-390, (2016).

[7] Jouan, Ph., Controllability of linear Systems on Lie group, Journal of Dynamics and Control Systems, Vol 17, pp. 591-616, (2011).

[8] Jouan, Ph. and Dath M., Controllability of linear systems on low dimensional nilpotent and solvable Lie groups, Journal of Dynamics and Control Systems, Vol 22, pp. 207-225, (2016).

[9] Jouan, Ph., Equivalence of control systems with linear systems on Lie groups and homogeneous spaces, ESAIM: Control Optimization and Calculus of Variations, Vol 16, pp. 956-973, (2010). 


\section{Víctor Ayala}

Instituto de Alta Investigación,

Universidad de Tarapacá

Casilla 7D,

Arica,

Chile

e-mail : vayala@ucn.cl

\section{Adriano Da Silva}

Instituto de Matemática

Universidade Estadual de Campinas

Cx. Postal 6065,

13.081-970 Campinas-SP,

Brasil

e-mail :

and

\section{Eyüp Kizil}

Instituto de Ciências Matemáticas e de Computação, Universidade de São Paulo, Cx. Postal 668,

CEP: 13.560-970,

São Carlos-SP,

Brasil

e-mail : kizil@icmc.usp.br 